\title{
Acute Superior Mesenteric Vein Thrombosis With Small Intestinal Ischemia
}

\author{
Kuldeep Singh
}

\begin{abstract}
Acute superior mesenteric vein thrombosis results in sudden interruption of blood supply to a segment of small intestine leading to ischemia followed by intestinal infarction and necrosis of that segment. Immediate systemic anticoagulation and early surgical intervention are required to remove the infracted segment of bowel to prevent sepsis and death. The mesenteric ischemia due to thrombosis of superior mesenteric vein is a rare condition of the intestine, however intestinal infarction associated with it is even more uncommon. Systemic hypercoagulable state is usually present in most of these patients. I, report a case of 43 years old Caucasian male who presented to the emergency room with severe diffuse upper abdominal and back pains out of proportion of his abdominal findings associated with nausea and vomiting. Past history of deep vein thrombosis (DVT) of lower extremity in the patient and his sister was documented. Computed tomography of abdomen with intravenous contrast revealed superior mesenteric vein thrombosis and extension of thrombus into portal vein along with its segmental branches resulting in ischemia of distal small bowel. The patient was initially treated with systemic anticoagulation but due to lack of response to conservative management for $12 \mathrm{~h}$ the patient was taken to operating room and underwent resection of about 2 feet of ischemic distal small bowel with good post operative recovery. Acute superior mesenteric vein thrombosis associated with mesenteric ischemia requires prompt diagnosis, urgent medical and early surgical intervention to prevent sepsis, high morbidity and mortality.
\end{abstract}

Keywords: Superior mesenteric vein thrombosis; Mesenteric ischemia; Computed tomography intestinal infarction; Deep vein thrombosis; Hypercoagulable state; Medical treatment; Anticoagulation; Surgical intervention

\section{Introduction}

Acute mesenteric ischemia was first described in 1895. It oc-

Manuscript submitted June 25, 2018, accepted July 20, 2018

Department of Emergency Medicine, UH Twinsburg Health Center, University Hospitals, Twinsburg, OH 44087, USA.

Email: drsinghmd@yahoo.com

doi: https://doi.org/10.14740/jmc3118w curs when the blood supply to the intestine is interrupted as a result of venous or arterial blockage of mesenteric vessels. It may be due to superior mesenteric vein thrombosis, non occlusive in a low flow state, arterial emboli or atherosclerotic arterial thrombosis. This process could be very acute, sub acute or chronic in nature and eventually lead to intestinal ischemia and necrosis. The incidence is very low $(0.09$ to $0.2 \%)$ of all acute emergency room abdominal pain visits [1]. Superior mesenteric vein thrombosis is uncommon and accounts for only $5-15 \%$ of all cases of acute mesenteric ischemia [2]. It usually involves small intestine but rarely may affect the large intestine also. Patient presents with acute severe generalized abdominal pain out of proportion to abdominal findings associated with nausea, vomiting, blood in stools but no weight loss. Hypercoagulability state, either hereditary or acquired usually exists in majority of these patients with mesenteric venous thrombosis. The treatment is comprised of systemic anticoagulation initially but early surgical intervention shall be contemplated to avoid high morbidity and mortality in conservative treatment failure. Computed tomography (CT) of abdomen with intravenous contrast is usually diagnostic in these conditions. I, report a case of acute superior mesenteric vein thrombosis with a history of deep vein thrombosis (DVT) of lower extremity treated in the past, family history of DVT of leg, presented to the emergency room with acute diffuse upper abdominal pain and back pain of few days duration but got worse for the last $8 \mathrm{~h}$. He was discovered to have thrombosis of superior mesenteric vein and portal vein on CT scan of abdomen with intravenous contrast. The patient was treated with intravenous heparin immediately but did not improve and later on underwent surgical resection of about 2 feet of infracted and necrotic segment of distal small bowel with good post operative recovery.

\section{Case Report}

A 43-year-old Caucasian male presented to a free standing emergency room in March, 2018 with acute diffuse upper abdominal pain (scale of 8 out of 10) and back pains for a few days associated with nausea and vomiting. The pain got worse for the last $8 \mathrm{~h}$ prior to visiting to the emergency room. He denied any blood in the stools or urinary symptoms. He contributed his back pain due to recent heavy lifting. There was significant past history of kidney stones, DVT of right lower extremity treated with coumadin for 6 months 7 years ago, history of seizure disorder taking Dilantin and $81 \mathrm{mg}$ of aspirin daily but 
not diabetic. His sister did also have history of DVT of left leg. His mother was diabetic. Abdominal examination was benign, soft, non distended, mild generalized discomfort but no evidence of acute abdomen; no acute tenderness, no guarding or rebound with faint bowel sounds present. Laboratory findings revealed WBC 15,100; hemoglobin of $16.5 \mathrm{~g}$, blood sugar 212, and lactate 1.6 (normal), coagulation profile was essentially normal, urine analysis normal. CT scan of abdomen with intravenous contrast confirmed complete thrombosis of superior mesenteric vein and its tributary branches but the thrombus also extended to portal vein and its branches. There was marked bowel wall thickening and stranding of multiple ileal loops but no evidence of pneumatosis intestinalis or portal venous gas. There was no free peritoneal air but mild pelvic fluid and mesenteric edema noted. The patient was admitted under surgical service with vascular surgery consult and immediately started on intravenous heparin. But due to continued severe abdominal pain with signs of peritoneal irritation and persistent leukocytosis, the patient was taken to operating room $12 \mathrm{~h}$ after admission. About 2 feet of ischemic distal small bowel was removed with uneventful post operative course and recovery. There was no perforation of the bowel noted during surgery or on pathological examination of the specimen. A second look operation was not necessary.

\section{Discussion}

Acute mesenteric ischemia is usually due to sudden mesenteric vein occlusion leading to decreased perfusion of an intestinal segment that may subsequently become ischemic; infarction and perforation of that segment of intestine may occur. It may lead to peritonitis, sepsis, organ failure and demise of the individual. Acute superior mesenteric vein thrombosis with small bowel ischemia is a very uncommon condition accounts for $5-15 \%$ of all cases of mesenteric ischemia [2]. Approximately $20-40 \%$ of patients has previous history of deep vein thrombosis or may have a pre-existing hereditary hypercoagulable state (protein $\mathrm{C}$ deficiency, protein $\mathrm{S}$ deficiency, anti thrombin III deficiency, factor V Leiden deficiency, or antiphospholipid syndrome [3]. Acquired conditions may also contribute to mesenteric venous thrombosis. It has been reported in certain cases of acute pancreatitis, laparoscopic sleeve gastrectomy for obesity [4], large abdominal tumors leading to venous compression, malignancy, viral infections, and myeloproliferative disorders. About 20 to 40percent of the cases are idiopathic [5]. Venous thrombosis usually is due to stagnation of venous blood flow, vessel wall injury, and hypercoagulability, called Virchow's triad.

Mesenteric vein thrombosis primarily involves superior mesenteric vein, and occasionally portal vein with or without acute ischemia of distal small bowel. But thrombosis does occur in the inferior mesenteric vein resulting in ischemia of the colonic segment [6]. Acute thrombotic process of mesenteric veins leads to decreased blood flow, increased venous pressure with exudation of fluid into the tissues causing marked bowel wall edema, sub mucosal hemorrhage and eventually bowel wall infarction with perforation. Finally sepsis, organ failure and death may ensue. However not all patients with mesenteric venous thrombosis are associated with intestinal ischemia or infarction. If the occlusion is gradual then a collateral circulation develops with no bowel wall ischemia. Chronic mesenteric vein thrombosis may lead to the development of significant collaterals with portal hypertension and bleeding [7]. Venous occlusion may cause arterial spasm in experimental studies and eventually lead to thrombosis and intestinal infarction [8]. Acute mesenteric ischemia may also occur in acute arterial occlusion and non occlusive low flow states. Arterial blockage could be acute or chronic in nature and it may be secondary to arterial emboli and atherosclerotic thrombotic event of celiac, superior and inferior mesenteric arteries.

A high index of suspicion in required to make the diagnosis of acute mesenteric vein thrombosis in patients who have severe colicky abdominal pain out of proportion of the clinical abdominal findings on examination. A comprehensive past and family history of thrombotic event or hereditary hypercoagulable state may provide additional help to make the diagnosis. In $50 \%$ cases a personal and family history of DVT of lower extremity or pulmonary embolism is present [9]. A definitive diagnosis of mesenteric vein thrombosis is established with imaging studies. CT of abdomen with intravenous and oral contrast is most reliable method to make the diagnosis. However CT abdomen angiography, MR abdomen angiography or catheter based angiography may be performed. CT abdomen findings may include venous filling defects, enhanced venous wall, enhanced bowel wall with edema, mesenteric stranding and edema, infarction of bowel wall with intestinal pneumatosis, portal venous gas, bowel dilatation or free peritoneal fluid. Time permitting hereditary or acquired hypercoagulable state should be excluded.

Treatment of acute mesenteric vein thrombosis is medical and or surgical. In early stages conservative management with systemic anticoagulation, hydration, bowel rest and serial clinical examination is adequate. However if the patient is unresponsive to conventional medical management and there is clinical, radiological or laboratory evidence of deterioration for suspected bowel infarction or necrosis a surgical exploration and bowel resection of the ischemic segment of the intestine shall be immediately performed. Open approach is preferred over laparoscopic method of abdominal exploration. Limited resection of the nonviable bowel with primary anastomosis may be performed in most cases. Second look operation may be necessary in some cases of questionable intestinal viability in $12-48 \mathrm{~h}$ [10]. Post operative long-term anticoagulation is usually recommended. In spite of early diagnosis and aggressive management the mortality rate is still very high i.e. $10-20 \%$ [11]. But mortality may be as high as $75 \%$ in intestinal infarction of a large segment of intestine. Recurrence of acute mesenteric vein thrombosis is low but it has been reported. Post operative small bowel stricture can occur in some cases.

The case presented here shares many commonalities of above discussion. The patient presented with severe abdominal pain out of proportion of the abdominal findings with past and family history of DVT of lower extremity was treated with systemic coagulation and surgical resection of infracted small bowel segment with uneventful postoperative recovery. The patient is doing well at present and receiving oral anticoagula- 
tion.

\section{Conflict of Interest}

No conflict of interest and no grant.

\section{References}

1. Stoney RJ, Cunningham CG. Acute mesenteric ischemia. Surgery. 1993;114(3):489-490.

2. Acosta S. Mesenteric ischemia. Curr Opin Crit Care. 2015;21(2):171-178.

3. Hertzberg MS, Underwood T, Favaloro EJ. Mesenteric vein thrombosis secondary to combined protein $\mathrm{C}$ deficiency and double heterozygosity for factor $\mathrm{V}$ Leiden and prothrombin G20210A. Am J Hematol. 1999;62(3):199200.

4. Villagran R, Smith G, Rodriguez W, Flores C, Cariaga M, Araya S, Yanez M, et al. Portomesenteric Vein Thrombosis After Laparoscopic Sleeve Gastrectomy: Incidence, Analysis and Follow-Up in 1236 Consecutive Cases.
Obes Surg. 2016;26(11):2555-2561.

5. Salvador SJ, Trerotola SO. Venous interventional radiology with clinical perspectives, 2nd. New York: Thieme, 2000; 13-15.

6. Clavien PA, Durig M, Harder F. Venous mesenteric infarction: a particular entity. Br J Surg. 1988;75(3):252255.

7. Harnik IG, Brandt LJ. Mesenteric venous thrombosis. Vasc Med. 2010;15(5):407-418.

8. Polk HC, Jr. Experimental mesenteric venous occlusion: 3. Diagnosis and treatment of induced mesenteric venous thrombosis. Ann Surg. 1966;163(3):432-444.

9. Harward TR, Green D, Bergan JJ, Rizzo RJ, Yao JS. Mesenteric venous thrombosis. J Vasc Surg. 1989;9(2):328333.

10. Khodadadi J, Rozencwajg J, Nacasch N, Schmidt B, Feuchtwanger MM. Mesenteric vein thrombosis. The importance of a second-look operation. Arch Surg. 1980;115(3):315-317.

11. Schoots IG, Koffeman GI, Legemate DA, Levi M, van Gulik TM. Systematic review of survival after acute mesenteric ischaemia according to disease aetiology. $\mathrm{Br} \mathrm{J}$ Surg. 2004;91(1):17-27. 